\title{
Freeze Drying as a Method to Produce Lyophilized Laban Immo: A Traditional Yogurt base Lebanese Recipe
}

\author{
Ossama Dimassi ${ }^{1 *}$, Hoda Saad Elmasri ${ }^{1}$, Mohammed Rached ${ }^{2}$, Raymond \\ Akiki $^{3}$, Ehsan Sharif-Askari ${ }^{2}$
}

\footnotetext{
${ }^{1}$ Department of Nutrition and Food Science, Lebanese International University, Beirut, Lebanon

${ }^{2}$ Department of Biomedical Sciences, Lebanese International University, Beirut, Lebanon

${ }^{3}$ Department of Business Administration, Lebanese International University, Beirut, Lebanon

*Corresponding authors: odimassi@gmail.com
}

\begin{abstract}
Laban immo (LI) is a traditional dish which mainly contains onions, meat, starch and yogurt. Based on a registered LI recipe four powdered products with Low Fat (LF) cow-, medium fat (MF) cow-, full fat (FF) cow-and FF goat-yogurt were produced and all were subjected to freeze-drying.

LI with FF- cow yogurt had the significantly lowest moisture loss (ML) but the significantly highest water activity (aw). LI powder with LF cow yogurt had the significantly lowest water activity, followed by that done with FF goat-, then with MF cow-and ended with FF cow-yogurt being the significantly highest. Concerning the pH, LI with FF goat yogurt had the significantly lowest value and the LI with MF cow yoghurt had the significantly highest value.

As for protein content LI with goat yogurt had the significantly lowest value compared to LI from cow yogurt. Concerning the ash content LI with goat yogurt had the significantly highest value compared to those with cow yogurt.

In this study freeze dried LI with full fat goat yogurt scored significantly the highest in the preference test followed by the LI with FF cow yogurt which was significantly higher than those with MF-and LF-cow yogurt. Triangle tests have shown that LI from natural yogurt and the reconstituted LI are sufficiently similar to be used interchangeably. Therefore, taste wise the production of LI in the powder form is possible and thus rendering it more convenient, easy to prepare and portable.
\end{abstract}

Keywords_Laban Immo, cooked yogurt, Moisture loss, water activity, preference test, triangle test.

\section{INTRODUCTION}

"Laban Immo" is one of the traditional Lebanese dishes containing many natural and healthy ingredients with yogurt as its base. It literally means "the milk of his mother". Laban Immo is a yogurt stew with lamb, onions and dried mint. This dish is consumed across the Eastern Mediterranean in Lebanon, Palestine (where it is called Tabeekh), Jordan, and Syria (where it is called Shakrieh). Because of the current fast-paced lives, less time is dedicated to the preparation of meals (Alkerwi, Crichton, $\&$ Hebert, 2015). Consequently, the consumption of ready meals (including ready-to-heat prepackaged dishes and fast food restaurant items) has increased. These ready meals present a quick and easy alternative to home prepared meals (Alkerwi et al., 2015).

Concerning yogurt consumption in Lebanon, the Lebanese market continues to have one of the highest per capita consumers of dairy products globally (Elie Haddad \& Chamoun, 2014). Supermarket shelves are increasingly stacked with dairy products with a significant increase in demand for goat yogurt; exceeding the available supply (Rahhal, 2018). Thus, presenting a traditional Lebanese yogurt based recipe in an easy-to-prepare format will have a great marketing potential internally and externally. The internal market is showing an increase in the consumption of convenient meals and yogurt based products constitutes a portion of this trend (Sharon Rady Rolfes, Kathryn Pinna, \& Ellie Whitney, 2015)

Compared to conventional food preservation technologies, the advantages of freeze drying include: retention of morphological, biochemical, and immunological properties, high viability/activity levels, lower temperature, and shear conditions compared with other drying methods, high recovery of volatiles, retention of structure, surface area, and stoichiometric ratios, high yield, long shelf life and reduced weight for storage, shipping, and handling (Jaya, 2009) (Koc, Yilmazer, Balkır, \& Ertekin, 2010). However, freeze 
drying is considered the most expensive operation for manufacturing a dehydrated product due to the high energy consumption and high costs of both operation and maintenance (Ciurzyńska \& Lenart, 2011).

Therefore, the potential of presenting this basic dish in a dried form that will make it handy, portable, with a long shelf life and a time-efficient-easy-cooking method (Santos, Nogueira, \& Rosenthal, 2018). Another aspect is the portion control. This form of food will allow for portion control by customizing packaging.

\section{MATERIAL AND METHOD}

\subsection{Sample Preparation}

Four recipes of laban immo (Table 1) were prepared using yogurt with 4 different fat contents: skim fat cow yogurt, medium fat cow yogurt, full fat cow yogurt, and full fat goat yogurt. All other ingredients remained the same in the 4 recipes (Zaarour, 2012).

Table 1 Four Laban Immo Recipes with different yogurts

\begin{tabular}{lcccc}
\hline INGREDIENT & $\begin{array}{c}\text { Reciepe 1 } \\
\text { SM yogurt } \\
\text { (grams) }\end{array}$ & $\begin{array}{c}\text { Reciepe 2 } \\
\text { MF Yogurt } \\
\text { (grams) }\end{array}$ & $\begin{array}{c}\text { Reciepe 3 } \\
\text { FF Yogurt } \\
\text { (grams) }\end{array}$ & $\begin{array}{c}\text { Reciepe 4 } \\
\text { FF Goat Yogurt } \\
\text { (grams) }\end{array}$ \\
\hline Oil & 6.5 & 6.5 & 6.5 & 6.5 \\
Lean Meat & 125 & 125 & 125 & 125 \\
Onions & 152.5 & 152.5 & 152.5 & 152.5 \\
Yogurt & 500 & 500 & 500 & 500 \\
Corn starch & 10 & 10 & 10 & 0 \\
Salt & 3 & 3 & 3 & 3 \\
Pepper & 0.25 & 0.25 & 0.25 & 0.25 \\
Dried Mint & 0.5 & 0.5 & 0.5 & 0.5 \\
Water & 126 & 126 & 126 & 0 \\
\hline TOTAL(grams) & 923.75 & 923.75 & 923.75 & 787.75 \\
\hline SM is Skim & &
\end{tabular}

SM is Skim milk; MF is Medium Fat; FF is Full Fat;

Laban immo was cooked to an internal temperature of $72^{\circ} \mathrm{C}$ for the lamb chops (Sharon Rady Rolfes et al., 2015). Then each recipe was homogenized using a mixer at medium speed for 7 minutes (Anang Catur Sulaksono, Sri Kumalaningsih, Wignyanto, \& Santoso, 2013). The paste were placed into $45 \mathrm{ml}$ conical tubes and lyophilized at $-40^{\circ} \mathrm{C}$ and $0.03 \mathrm{~m}$ Tor pressure by Boyikang vacuum freeze dryer. For sensory analysis the freeze dried sample were reconstituted to their original moisture content.

\subsection{Methods}

Moisture content: Drying Oven and Balance method was used for moisture content determination. The oven used was Contherm designer series (Contherm Scientific LTD) following the IS 11623: 1986.

Moisture Loss: Moisture loss was determined by subtracting the weight of the sample after freeze drying, where no loss was detected anymore, from the weight of the sample before freeze drying using the following equation Eq. (1).

$\%$ moisture $\operatorname{loss}=[$ (initial weight-final weight $) /$ initial weight]*100

Weight determination: Weight was measured using Analytical Balance MS304TS/00 with 320 g capacity and $0.1 \mathrm{mg}$ readability.

pH analysis: Microcomputer based $\mathrm{pH} /$ conductivity/TDS/salinity and temperature pocket meter Model $\mathrm{pH} / \mathrm{EC} 80$ was used to measure the $\mathrm{pH}$ (Jenco VisionP).

Water activity: It was determined using Pawkit water activity meter. Sample were flattened to cover the bottom of the cup and then water activity was measured at room temperature (Suzann, 2010).

Fat Determination: The fat content was determined using Soxhlet method as described by AOAC 922.06.

Protein Determination: Protein content was determined using the Kjeldahl method according to AOAC 991.20.

Ash Determination: Ash was determined using the AOAC 942.05 method.

Powder Production: The samples were lyophilized by Boyikang vacuum freeze dryer.

\subsection{Statistical analysis}

All tests and analysis were run in triplicates and averaged. General linear repeated measure model performed via SPSS (statistical Package for the Social Sciences, version 17.0) was used to study the difference in the physicochemical properties of the powder produced from the different yogurt recipes (moisture content, water activity, $\mathrm{pH}$, protein, fat and ash content) (Kwanchai A. Gomez \& Gomez, 1984).

Furthermore, chi square was used to compare the preference of the four different recipes (ranking). In addition to that, triangle test was done to evaluate if differences would be detected between the freshly cooked recipe and its reconstituted counterpart.

\section{RESULTS}

3.1. Moisture Content Moisture loss and water activity of the four laban immo recipes

Starting with the moisture loss the full fat cow yogurt laban immo powder was the significantly lowest, while those of the skim milk cow, medium fat cow and full fat goat yogurt did not differ significantly (Fig.1). 


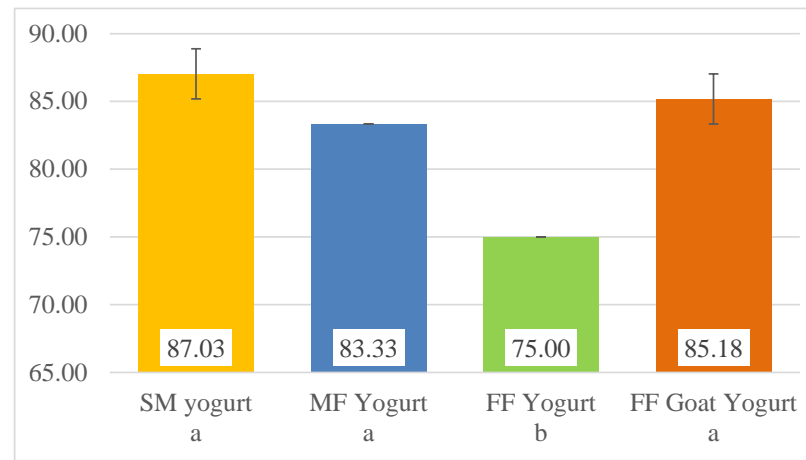

Fig.1: Percent Moisture loss of powder from the four laban immo reciepes

SM is Skim milk; MF is Medium Fat; FF is Full Fat;

The moisture content the laban immo powder done with skim milk cow yogurt was significantly the lowest followed by the moisture content of laban immo powder done with the full fat cow yogurt which did differ significantly from the moisture content of laban immo powder done from those done with full fat goat yogurt and medium fat cow yogurt being significantly the highest (Table 2).

Concerning the water activity, all powders of laban immo done with the different yogurts did differ significantly from each other with that done with the skim milk cow yogurt being the significantly lowest, followed by the water activity of laban immo done with full fat goat yogurt, then by those done with medium fat cow yogurt. Furthermore, the water activity of laban immo powder done by full fat cow yogurt was significantly the highest (Table 2).

Table 2 Moisture content and water activity of the fourlaban immo reciepies

\begin{tabular}{|c|c|c|c|c|}
\hline Yogurt Used & \multicolumn{2}{|c|}{ Moisture } & \multicolumn{2}{c|}{ Water Activity } \\
\cline { 2 - 5 } In Laban Immo Recipe & \multicolumn{2}{|c|}{ Mean Std. Error } & \multicolumn{1}{c|}{ Mean } & \multicolumn{2}{c|}{ Std. Error } \\
\hline SM yogurt & $29.10 \mathrm{a}$ & 1.85 & $0.18 \mathrm{a}$ & 0.02 \\
\hline MF Yogurt & $55.37 \mathrm{~b}$ & 0.00 & $0.46 \mathrm{~b}$ & 0.01 \\
\hline FF Yogurt & $40.73 \mathrm{c}$ & 0.00 & $0.61 \mathrm{c}$ & 0.02 \\
\hline FF Goat Yogurt & $50.97 \mathrm{~b}$ & 1.85 & $0.38 \mathrm{~d}$ & 0.01 \\
\hline Average & 44.04 & 1.49 & 0.41 & 0.02 \\
\hline
\end{tabular}

Numbers with different letter are significantly different

SM is Skim milk; MF is Medium Fat; FF is Full Fat;

3.2. Protein and fat content of the laban immo powder.

There is no significant difference between the protein values of the laban immo powder done from the skim milk, medium fat and full fat cow yogurt, which were significantly higher than the laban immo powder done using full fat goat yogurt (Table 3).
As for the fat content, as expected, all powder did differ significantly from each other with laban immo powder from skim milk cow yogurt being the lowest and the powder done from the full fat goat yogurt being significantly the highest.

Table 3 Protein and fat content of powder from the fourlaban immo reciepies

\begin{tabular}{|c|c|c|r|c|}
\hline Yogurt Used & \multicolumn{2}{|c|}{ Protein } & \multicolumn{2}{c|}{ Fat } \\
\cline { 2 - 5 } In Laban Immo Recipe & \multicolumn{2}{|c|}{ Mean Std. Error } & Mean & \multicolumn{2}{|c|}{ Std. Error } \\
\hline SM yogurt & $44.76 \mathrm{a}$ & 0.48 & $0.95 \mathrm{a}$ & 0.19 \\
\hline MF Yogurt & $46.83 \mathrm{a}$ & 0.26 & $6.63 \mathrm{~b}$ & 0.14 \\
\hline FF Yogurt & $45.58 \mathrm{a}$ & 0.65 & $7.79 \mathrm{c}$ & 0.59 \\
\hline FF Goat Yogurt & $39.41 \mathrm{~b}$ & 1.55 & $12.48 \mathrm{~d}$ & 0.18 \\
\hline Average & 44.14 & 0.93 & 6.96 & 1.25 \\
\hline
\end{tabular}

Numbers with different letter are significantly different $\mathrm{SM}$ is Skim milk; MF is Medium Fat; FF is Full Fat;

3.3. Ash content and $\mathrm{pH}$ of powder from the fourlaban immo recipes.

The ash content and the acidity of laban immo powder done with the full fat goat yogurt were significantly the highest with those of the other powders being more comparable (Table 4).

Table 4 Ash content and pH of the four-laban immo recipes

\begin{tabular}{|c|r|c|r|c|}
\hline Yogurt Used & \multicolumn{2}{|c|}{ Ash } & \multicolumn{2}{c|}{ PH } \\
\cline { 2 - 5 } In Laban Immo Recipe & Mean Std. Error & Mean & \multicolumn{2}{|c|}{ Std. Error } \\
\hline SM yogurt & $2.09 \mathrm{a}$ & 0.06 & $5.03 \mathrm{a}$ & 0.04 \\
\hline MF Yogurt & $3.68 \mathrm{~b}$ & 0.16 & $5.15 \mathrm{~b}$ & 0.03 \\
\hline FF Yogurt & $2.14 \mathrm{a}$ & 0.03 & $4.93 \mathrm{a}$ & 0.03 \\
\hline FF Goat Yogurt & $4.29 \mathrm{c}$ & 0.03 & $4.66 \mathrm{c}$ & 0.04 \\
\hline Average & 3.05 & 0.29 & 4.94 & 0.06 \\
\hline
\end{tabular}

Numbers with different letter are significantly different SM is Skim milk; MF is Medium Fat; FF is Full Fat;

\subsection{Sensory analysis.}

Rank preference test was performed using 30 test subjects in order to rank samples from the 4 recipes according to preference. Furthermore, the preferred recipe was subjected was subjected to delta.

\subsubsection{Results of preference ranking test}

The laban immo done using full fat goat yogurt different significantly from the laban immo done with full fat cows yogurt, which in turn differ significantly from laban immo done with medium fat- and skim milk- cows yogurt which did not differ significantly (Table 5). Furthermore, the laban immo done with the goat yogurt was chosen as rank one the most (Table 5). Furthermore, the laban immo done with the full fat cows yogurt was chosen as rank 2 the most (Table 5). Last but not least laban immo done 
with medium fat- and skim milk- cows yogurt were chosen as rank 3 and 4 the most.

Table 5 Preference test results of different laban Immo recipes

\begin{tabular}{ccccc}
\hline & $\begin{array}{c}\text { SM yogurt } \\
\text { a }\end{array}$ & $\begin{array}{c}\text { MF Yogurt } \\
\text { a }\end{array}$ & $\begin{array}{c}\text { FF Yogurt } \\
\text { b }\end{array}$ & $\begin{array}{c}\text { FF Goat Yogurt } \\
\text { c }\end{array}$ \\
\hline rank 1 & 3 & 1 & 4 & 22 \\
rank 2 & 4 & 5 & 19 & 2 \\
rank 3 & 12 & 14 & 1 & 3 \\
rank 4 & 11 & 10 & 6 & 3 \\
\hline SM
\end{tabular}

SM is Skim milk; MF is Medium Fat; FF is Full Fat;

\subsubsection{Results of Delta test}

The number of the correct responses and the number of the total responses were counted then compared to the table determine their statistical significance (Meilgaard, Civille, \& Carr, 2016). There was no significant difference between reconstituted the lyophilized freeze dried sample and that of the freshly cooked and served laban immo recipe.

\section{DISCUSSION}

Concerning the moisture content, percent moisture loss and water activity, the results show the importance of the water activity measurement as the real indicator of the different chemical reactions that might occur in the product (FU, TAOUKIS, \& LABUZA, 1991). While there was no significant difference in the percent moisture loss except for the powdered laban immo done with the full fat cow's milk, the moisture content showed a different story with the powdered laban immo done with the skim milk cows yogurt had the lowest moisture content and the laban immo powder done with the medium fat cows yogurt and full fat goat yogurt had the highest moisture content. This is turn would have been deceiving if the water activity have not been measured, where the labban immo done with the skim milk cows yogurt, as expected, had the lowest water activity but the powdered laban immo done with full fat cows yogurt had the significantly highest water activity. Although the laban immo done with full fat cow's milk had the lowest moisture content the fat also trapped the water and prevented it from evaporation. Concerning the powder laban immo done with full fat goat milk, the fat and the protein components are of different nature than that of cow's milk, where the fat globules are more naturally homogenized and the casein compnents (alfa s1, alfa s2, beta and kappa casein) differ from those of cow's milk (O. Dimassi, Hinrichs, \& Zárate, 2006) (Ossama Dimassi et al., 2005) (Clark \& W. Sherbon, 2000).
The protein content of all the laban immo powder from yogurt of cow's origin did not differ much from each other but that done from goat yogurt was significantly lower. This might partially explain why the water activity of the laban immo powder from the full fat goat yogurt was lower than that done with the full fat cows' yogurt although it had the significantly highest fat content.

Furthermore, the mineral dissociation and hydration of milk proteins especially the casein the extent of mineral depends on the $\mathrm{pH}$ value. At $\mathrm{pH}$ 5.2, like that of the laban immo powder done from medium fat cow yogurt, a part of calcium and the totality of the inorganic phosphate are solubilized. The nearer the $\mathrm{pH}$ to the 4.6 value, such as the $\mathrm{pH}$ of the laban immo powder from full fat goat yogurt, the more the solubilized (Le Graet \& Brulé, 1993) (Mekmene, Le Graet, \& Gaucheron, 2010). Added to that the casein micelles nearing the iso-electric point 4.6 is less charged thus less water would be bound to them (Graet \& le Gaucheron, 1999). This is another factor that would explain the water activity of the full fat goat compared to that of the powder done from the full fat cow's yogurt.

As for the preference ranking test the laban immo done from the full fat goat yogurt scored the highest followed by the laban immo done with the full fat cows yogurt and the goat recipe had the highest fat content. The later scored higher than the laban immo from the medium fat and skim milk yogurt which were significantly lower in fat percent. Although fat in itself is neutral it absorb lots of volatiles and thus the flavors are more conserved.

Furthermore, by comparing the reconstituted laban immo meal and that of fresh one no difference was detected. This gives this method a huge potential for the production of lyophilized laban immo product rendering it easier to store and prepare and most importantly easier to market and thus increasing the yogurt based products diversity, which in turn would help the dairy sector. Care, however, should be taken for the water activity which might be modified by simple additions or changing the drying method which needs further studies.

\section{CONCLUSION}

The results of this study suggested the yogurt from skim milk is the easiest to dry yielding the lowest water activity. Furthermore, there are differences between the recipe based on yogurt of the bovine and that of the caprine. Thus, more studies should be conducted to capture this difference. Furthermore, this traditional dish is already known in the Middle Eastern area thus it already has a potential market. Furthermore, it would open new possibilities of marketing in new arena. 


\section{ACKNOWLEDGEMENTS}

Special thanks to the dairy facility at "Al Akhdar Dairy" in Saida for making his fresh products available for us.

\section{REFERENCES}

[1] Alkerwi, A., Crichton, G. E., \& Hebert, J. R. (2015). Consumption of ready-made meals and increased risk of obesity: findings from the Observation of Cardiovascular Risk Factors in Luxembourg (ORISCAV-LUX) study. $\mathrm{Br}$ J Nutr, 113(2), 270-277. doi:10.1017/s0007114514003468

[2] Anang Catur Sulaksono, Sri Kumalaningsih, Wigny anto, \& Santoso, I. (2013). Production and Processing of Yoghurt Powder Using Foam-Mat Drying. Food and Public Health, 3(5), 235-239.

[3] Ciurzyńska, A., \& Lenart, A. (2011). Freeze-Drying Application in Food Processing and Biotechnology - a Review. Polish Journal of Food and Nutrition Sciences, 61(3), 165-171. doi:10.2478/v10222-011-0017-5

[4] Clark, S., \& W. Sherbon, J. (2000). Alpha s1-casein, milk composition and coagulation properties of goat milk (Vol. $38)$.

[5] Dimassi, O., Hinrichs, J., \& Zárate, A. V. (2006). Cheese production potential of milk from Dahlem Cashmere goats using a cheese simulation method. Small Ruminant Research, 65(1-2), 38-43. doi:10.1016/j.smallrumres.2005.05.035

[6] Dimassi, O., Neidhart, S., Carle, R., Mertz, L., Migliore, G., Mané-Bielfeldt, A., \& Valle Zárate, A. (2005). Cheese production potential of milk of Dahlem Cashmere goats from a rheological point of view (Vol. 57).

[7] Elie Haddad, \& Chamoun, N. (2014). Developing the typical dairy products of the Bekaa andBaalbeck-Hermel, Diagnosis and local strategy. Retrieved from

[8] FU, B., TAOUKIS, P. S., \& LABUZA, T. P. (1991). Predictive Microbiology for Monitoring Spoilage of Dairy Products with Time-Temperature Integrators. 56(5), 12091215. doi:10.1111/j.1365-2621.1991.tb04736.x

[9] Graet, Y., \& le Gaucheron, F. (1999). pH-induced solubilization of minerals from casein micelles: influence of casein concentration and ionic strength. AGRIS, 66(2), 215- 224.

[10] Jaya, S. (2009). Microstructure Analy sis of Dried Yogurt: Effect of Different Drying Methods. International Journal of Food Properties, 12(3), 469-481. doi:10.1080/10942910701772071

[11] Koc, B., Yilmazer, M. S., Balkır, P., \& Ertekin, F. K. (2010). Spray Drying of Yogurt: Optimization of Process Conditions for Improving Viability and Other Quality Attributes. Drying Technology, 28(4), 495-507. doi:10.1080/07373931003613809

[12] Kwanchai A. Gomez, \& Gomez, A. A. (1984). Statistical Procedures for Agricultural Research, (2nd Edition ed.).

[13] Le Graet, Y., \& Brulé, G. (1993). Les équilibres minéraux du lait: Influence du $\mathrm{pH}$ et de la force ionique (Vol. 73).

[14] Meilgaard, M. C., Civille, G. V., \& Carr, B. T. (2016). Sensory evaluation techniques.
[15] Mekmene, O., Le Graet, Y., \& Gaucheron, F. (2010). Theoretical model for calculating ionic equilibria in milk as a function of $\mathrm{pH}$ : comparison to experiment. J Agric Food Chem, 58(7), 4440-4447. doi:10.1021/jf903628r

[16] Rahhal, N. (2018, April). Say (goat) cheese. Lebanese dairy producers diversify into new opportunities. Exclusive Magasine, April.

[17] Santos, G. d., Nogueira, R. I., \& Rosenthal, A. (2018). Powdered yoghurt produced by spray drying and freeze drying: a review \%J Brazilian Journal of Food Technology. 21. Retrieved from http://www.scielo.br/scielo.php?script=sci arttext\&pid=S 1981-67232018000100307\&nrm=iso

[18] Sharon Rady Rolfes, Kathryn Pinna, \& Ellie Whitney. (2015). Understanding Normal and Clinical Nutrition, 10th Editio.

[19] Suzann, N. (2010). Food Analysis: Springer.

[20] Zaarour, M. b. (2012). So7i Wa Sari3 - (Arabic Edition) (Vol. November): Librairie du Moyen Orient. 